\title{
Using Pseudo-ZTs for Thermoelectric Materials Search
}

\author{
Shuang Tang*
}

Thermoelectrics is attracting intensive research interests. The band structure information of new compounds is growing rapidly with the power of first principle calculations. However, not every thermoelectric materials candidate can be experimentally investigated due to limited resources. Therefore, we need to develop an efficient approach of screening and selecting the most promising ones from the various band structure databases of new compounds. Here we propose the concept of pseudo-ZTs: $z t_{e}$ and $z t_{L}$, where $z t_{e}$ only measures the electronic influence and $z t_{L}$ only scales the lattice contribution. Using $z t_{e}$ and $z t_{L}$ helps avoid playing the "seesaw balancing game" between the Seebeck coefficient and the conductivities. It also helps reveal deeper physics on how dimensionality, carrier concentration and band structure will affect $Z T$ quantitatively. A range of thermoelectric materials are tested, and $\sim 400$ new compounds are calculated for predictions. The pseudo-ZTs can serve as a good guidance for thermoelectric materials search, in addition to the semi-empirical " $\beta_{S E}$ " indicator.

Keywords: Thermoelectrics; Figure-of-Merit;ZT; Psuedo-ZT; Low-Dimension; Band Structure; Carrier Concentration

Received 21 February 2019, Accepted 27 March 2019

DOI: $10.30919 / \mathrm{esmm} 5 \mathrm{f} 213$

\section{Broader Context}

Energy crisis is an urgent global problem economically, politically, and environmentally. To address such energy crisis, we have to develop high-efficiency approaches to use clean and renewable energy, e.g. waste heat, geothermal, nuclear, and solar energy. Since all the heatrelated energy resources, both fossil and green, have thermal processes and waste heat recycling problems, thermoelectric power generation is very attractive. Towards the direct high-efficiency conversion between heat flow and electricity, enhancing the thermoelectric figure-of-merit (ZT) is a complex problem and a research topic of broad interest. Modern first-principle computations have strong power in band structure prediction for new compounds, though transport calculations are still challenging and expensive. Due to limited resources, not every materials candidate can be experimentally investigated. Therefore, it will be very helpful to develop an efficient thermoelectric indicator based on band structure information to search for the most promising candidates from the various band structure databases. We here propose a framework of pseudo-ZTs to accelerate this task.

Thermoelectric materials offer a way to interconvert heat flow and electricity. The conversion efficiency is characterized by the material specified dimensionless figure-of-merit $Z T=\sigma S^{2} T / \kappa$, where $\sigma, S, T$ and $\kappa$ are the electrical conductivity, Seebeck coefficient, temperature and thermal conductivity, respectively. Until the past two decades, it was believed that $Z T$ could not exceed 1.' More recently, many novel approaches have sequentially pushed the upper limit of $Z T{ }^{2-8}$ including the utilization of low-dimensionalization ${ }^{9}$, sharp density of states ${ }^{10}$, superlattice, ${ }^{11-13}$ resonant states, ${ }^{14,}{ }^{15}$ nanocomposites, ${ }^{16,}, 17$ and pipe-shaped Fermi surfaces. ${ }^{18}$ Despite these progresses, values of $Z T$ still remain too

College of Engineering, State University of New York Polytechnic Institute, Albany, NY, USA

*E-mail: tangs1@sunypoly.edu low for inexpensive materials to consider the prevalent use of thermoelectrics. The strong correlation between $\sigma, S$ and $\kappa$ results in significant difficulties on this optimization problem. For example, it is typical that materials with large $\sigma$ always tend to have large $\kappa$ and small $S$, which kills $Z T$.

The recent advancement of first-principle calculations has enabled the prediction of band structures of a largely growing number of new compound materials, ${ }^{19}$ the information of which are collected in various materials databases. ${ }^{20-22}$ It is resource-prohibited to make efforts on each of these materials. Therefore, how to choose the most promising candidates from these databases for further improvement with experimental efforts is a crucial and urgent question.

To achieve this end, the semi-empirical thermoelectric indicator " $\beta_{S E}$ " was developed, ${ }^{23-25}$ which requires the input of estimated carrier mobility $(\mu)$ and lattice thermal conductivity $\left(\kappa_{L}\right)$. However, the absolute values of $\mu$ and $\kappa_{L}$ are difficult to predict, which can change substantially with crystal imperfections, such as the grain size, ${ }^{26}$ defects concentration, ${ }^{27}$ and grain boundary thickness. ${ }^{28}$ Although accurate values of $\kappa_{L}$ can be computed for several individual materials with expensive calculations, ${ }^{29-31}$ the general discrepancies between theoretical calculations and experimental measurements on thermal conductivity for most materials are still obvious. Further, $\kappa_{L}$ can be reduced significantly by novel techniques like nanocomposites synthesis. ${ }^{16,17}$ Thus, the band structure is still the most important input for the materials search before experimental investments.

Therefore, we here develop a framework of pseudo-ZTs to indicate the potential of good thermoelectric behavior. The pseudo-ZT prediction only needs to input the information of band structures. Further, we will see that the pseudo-ZTs can provide us with a more physical insight onto how the energy sensitivity of transport, the dimensionality, the band asymmetry, and the band gap will influence the $Z T$ quantitatively, and suggest strategies to further improve $Z T$. Therefore, the pseudo- $Z T$ system we developed here can be used along with the semi-empirical thermoelectric indicator " $\beta_{S E}$ " ${ }^{25}$ for searching potential thermoelectric materials among the numerous candidates. 
We see that using the formula of $Z T=\sigma S^{2} T / \kappa$, we are not allowed to change any one single variable $(\sigma, S$ or $\kappa)$ with the other two fixed, e.g. it is unable to enhance $Z T$ by increasing $\sigma$ and keeping $S$ and $\kappa$ unchanged, because $\sigma, S$ and $\kappa$ are highly correlated. Life will be much easier if we can find a way to decompose $Z T$ into quantities that are independent or weakly correlated to each other. To achieve this goal, we introduce the idea of splitting $Z T$ into two pseudo-ZTs: $z t_{e}$ and $z t_{L}$,

$$
\frac{1}{Z T}=\frac{1}{z t_{e}}+\frac{\kappa_{L}}{k_{B}^{2} T \theta} \frac{1}{z t_{L}}
$$

where $z t_{e}=\left(J_{0} J_{2} J_{1}^{-2}-1\right)^{-1}$ and $z t_{L}=J_{1}^{2} J_{0}^{-1}$. The $J_{n}^{\prime}$ s are dimensionless numbers defined as

$$
J_{n}=\frac{1}{\theta} \int\left(-\frac{\partial f_{0}}{\partial \varepsilon}\right) \Xi(\varepsilon)\left(\varepsilon-\varepsilon_{f}\right)^{n} d \varepsilon
$$

where $\varepsilon$ and $\varepsilon_{f}$ are the reduced carrier energy $\left(\varepsilon=E / k_{B} T\right)$, and reduced Fermi level $\left(\varepsilon_{f}=E / k_{B} T\right), f_{0}$ is the Fermi distribution, and $\Xi(\varepsilon)$ is the transport distribution as a function of $\varepsilon{ }^{32,33}$ Further, $\theta=\Xi(1)$ is a measure of the transport strength despite of its sensitivity to carrier energy. Thus, we see that $z t_{e}$ only measures the influence of electronic affection to $Z T$, while $z t_{L}$ only scales the contribution of the lattice thermal conductivity $\left(\kappa_{L}\right)$ to $Z T$. Just like $Z T, z t_{e}$ and $z t_{L}$ are also dimensionless, which we name as pseudo-ZTs. By using the much less correlated $z t_{e}$ and $z t_{L}$, we can avoid playing the "seesaw balancing game" between $S$ and $\sigma$ or $\sigma$ and $1 / \kappa$, when examining the possible enhancement of $Z T$ over different parameters, as shown in the following contents.

First, we use the pseudo-ZTs to examine the dimensionality paradox of thermoelectrics. Since Hicks and Dresselhaus proposed that low-dimensionality will benefit thermoelectric $Z T$, great efforts have been made in various nanoscale materials. ${ }^{34,35}$ It is observed that the lowdimensionalization strategy works in many but not all materials systems. ${ }^{36}$ We still have many cases where the low-dimensional systems have non-enhanced $Z T^{36}$ compared with their bulk counterparts. Thus, it is interesting to study where this discrepancy come from, and how we can distinguish the low-dimensionality favored materials from the nonfavored ones.

Here we try to study this problem from the relation between the pseudo-ZTs and the carrier energy sensitivity of transport. The carrier energy sensitivity of transport can be characterized by

$$
s=\left\langle\frac{d \Xi / \Xi}{d \varepsilon / \varepsilon}\right\rangle
$$

which is generally a function of carrier energy, temperature and band valley structures. For a single carrier, it is a specific value, while for the collective behavior of multiple carriers, it is a statistical measurement of the whole system. Such statistical measurements are popularly used in diffusive transport, e.g. the carrier mobility $\mu$ is a statistical measurement on how mobile the carriers are under an external field. The overall energy sensitivity will decrease upon low-dimensionalization, because (1) the density of states will have a smaller or even negative dependence to energy, and (2) the scattering time for high energy carriers encountering possible ballistic scatterings will have negative energy dependence $(\tau \sim \lambda \nu)$.

Fig. 1 (a) shows the pseudo-ZTs as a function of carrier concentration for different values of energy sensitivity $s$. We can see that decreased $s$ leads to increased $z t_{\mathrm{L}}$ but decreased $z t_{e}$. Equation (1) implies that the ultimate $Z T$ is dominated by the smaller pseudo- $Z T$. Thus, for high carrier concentration systems, $Z T$ is dominated by $z t_{e}$, while for low carrier concentration systems, $Z T$ is dominated by $z t_{L}$, according to Fig. 1 (a). Therefore, the conclusion is that the ultimate $Z T$ will be enhanced by low-dimensionalization only for systems with low carrier concentrations. For systems with high carrier concentrations, staying in the bulk form will be preferred. For systems with moderate carrier concentrations, the dimension of the material may not affect $Z T$ significantly.

To have a better understanding of the connection between the pseudo-ZTs and the $\beta_{S E}$ indicator, ${ }^{25}$ we illustrate their relation with carrier (a)

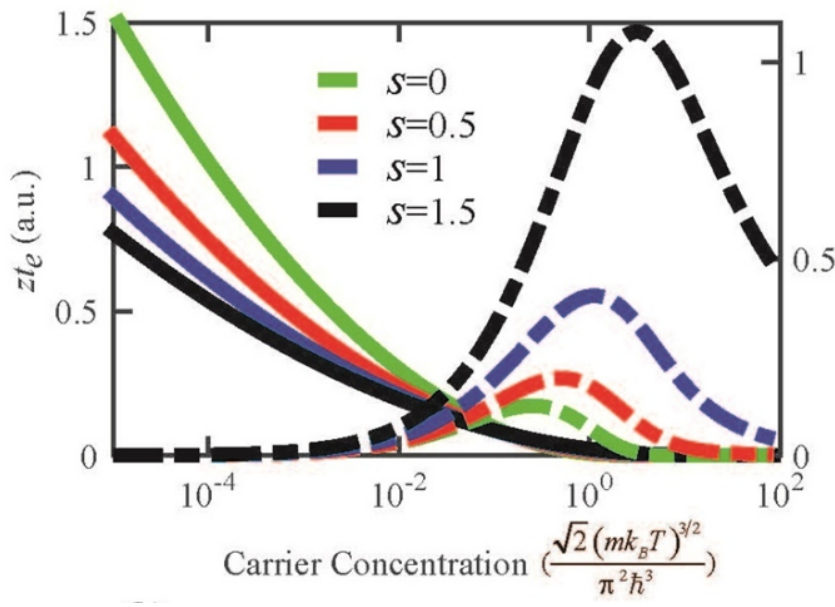

(b)

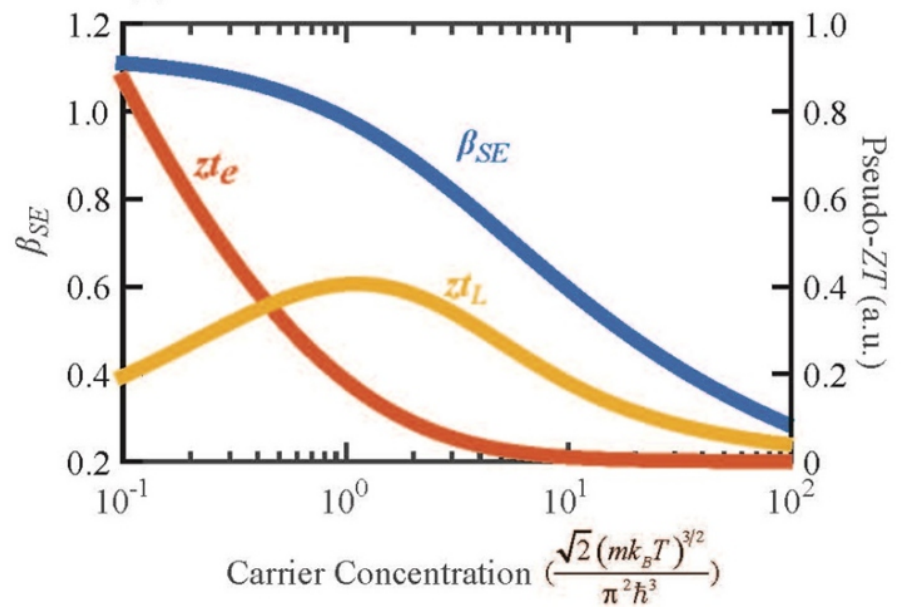

Fig. 1 (a) shows the pseudo-ZTs as a function of carrier concentration for different values of the energy sensitivity $s$. The colored solid (dashed) curves represent the values of $z t_{\mathrm{e}}\left(z t_{L}\right)$ for cases with different energy sensitivities $(s)$. The change of $s$ affect the two pseudo-ZTs, $z t_{\mathrm{e}}$ and $z t_{L}$, in opposite trends. Since the smaller pseudo-ZT dominates the overall $Z T$ as implied from Equation (1), $z t_{\mathrm{L}}$ and $z t_{e}$ dominates in low and high carrier concentration regimes, respectively. Therefore, the low-dimensionalization approach can only enhances $Z T$ significantly in low carrier concentration materials systems. (b) The relation between the pseudo-ZTs and the $\beta_{S E}$ indicator, ${ }^{25}$ illustrated for the $s=1.0$ case as an example. It can be seen that the $\beta_{S E}$ indicator can only well estimate $Z T$ for the high carrier concentration regime. For the low carrier concentration regime, the dominant pseudo-ZT $\left(z t_{L}\right)$ has an opposite trend with the $\beta_{S E}$ indicator vs. carrier concentration. Therefore, the $\beta_{S E}$ indicator in this regime is no longer a good estimation. Without loss of generality and validity, the single valley calculation and the arbitrary unit are used for the simplicity and convenience of illustration. 
concentration in Fig. 1 (b). We can see that the $\beta_{S E}$ indicator can well estimate $Z T$ for the high carrier concentration region. However, when the carrier concentration is low, the dominant pseudo-ZT becomes $z t_{L}$ that has an opposite trend compared to the $\beta_{S E}$ indicator as a function of carrier concentration. Therefore, the $\beta_{S E}$ indicator in this region is no longer a good estimation.

Second, we use the pseudo-ZTs to give us a guidance for screening and selecting untested candidates from the databases of band structures. ${ }^{20-22}$ We will examine the most important properties of a band structure, including band asymmetry, band gap, and band-edge alignment. Qualitative comments on how these properties may affect $Z T$ might be found occasionally in the literature. ${ }^{37}$ Here a quantitative approach on how to comprehensively evaluate a material is provided based on the pseudo-ZT concept.

The band asymmetry characterizes how much the valence and the conduction bands are different from each other. While the shape of band edges have to be considered case by case for high accuracy $Z T$ calculations, especially for the irregular shaped band structures, the ratio $(\gamma)$ between the density-of-state masses of each band can be used as a primary feature parameter for the efficient materials search purpose. Fig. 2 has shown how the maximum values of $z t_{e}$ and $z t_{L}$ will be affected by $\gamma$. Without loss of generality, we illustrate a case of reduced band gap $\varepsilon_{g}=20$. We see that unlike the case of $s$ in Fig. 1, $\gamma$ is a parameter that can increase both $z t_{e}$ and $z t_{L}$ together. In other words, the task of enhancing $Z T$ always prefers holes and electrons to be as different from each other as possible. This explains why materials that can provide large band asymmetry with both heavy holes and light electrons, such as topological insulator, ${ }^{38,}{ }^{39}$ quasi-Dirac-cones, ${ }^{40,}{ }^{41}$ or narrow-band-gaps, ${ }^{42}$ are always good thermoelectric materials. This also explains why several nano-composites systems have been reported to have high $Z T^{16,}$

${ }^{17}$ wherein the holes-screening grain-boundaries are effectively making holes much heavier than electrons, resulting in large $\gamma$ values.

We have found that in contrary to conventional thought, a large band gap can enhance both pseudo-ZTs, until the band gap becomes highly correlated with lattice thermal conductivity. Our result is different from Ref. 32, where only the $z t_{L}$ dominated systems are considered. To illustrate this, we use a hypothetic system with a fixed $\gamma$, so we can examine $z t_{e}$ and $z t_{L}$ as a function of $\varepsilon_{g}$. Without loss of generality, we illustrate a case where $\gamma=1$, as shown in Fig. 3. Fig. 3 has dissolved the traditional "fear" of large band gap, which implies small carrier concentration. It clearly shows that the maximum values of both pseudo-ZTs will increase or at least not decrease with $\varepsilon_{g}$. However, it is worth to note that the carrier concentration of large band gap insulators could become difficult to tune through doping. Furthermore, a large band gap may be positively correlated with the thermal conductivity, i.e. large band gap insulators usually have long inter-atomic distances that result in large lattice thermal conductivity, which compromises the enhanced $z t_{L}$ value.

For a multi-valley or irregular-shaped band structure that can be decomposed into a series of small parabolic or non-parabolic valleys. The shape similarities and energy degeneracy can also be quantitatively evaluated by the pseudo-ZTs. Here we illustrate this approach by comparing a one-valley band and a two-valley band. For more complex band structures, this approach can be similarly employed. For instance, assuming that we have two hypothetic band valleys denoted as $\mathrm{V}^{(1)}$ and $\mathrm{V}^{(2)}$. We use the ratio $(\zeta)$ between the curvatures of $\mathrm{V}^{(1)}$ and $\mathrm{V}^{(2)}$ as the primary feature parameter to characterize their shape similarity, and the difference in energy at the bottoms of $\mathrm{V}^{(1)}$ and $\mathrm{V}^{(2)}$, i.e. $\Delta E=\Delta \varepsilon \cdot k_{B} T$, to characterize the degree of degeneracy between the two valleys. $V^{(1)}$ and $\mathrm{V}^{(2)}$ are fully degenerated when $\Delta \varepsilon=0$. Further, we use the optimization ratio to compare the maximum pseudo-ZTs in different cases, defined as,

$$
\text { Optimization Ratio }=\frac{z t_{\max }^{(1)+(2)}}{\max \left[z t_{\max }^{(1)}, z t_{\max }^{(2)}\right]}
$$

where the pseudo-ZTs denoted by a lower case $z t$ stands for $z t_{e}$ or $z t_{L}$, the

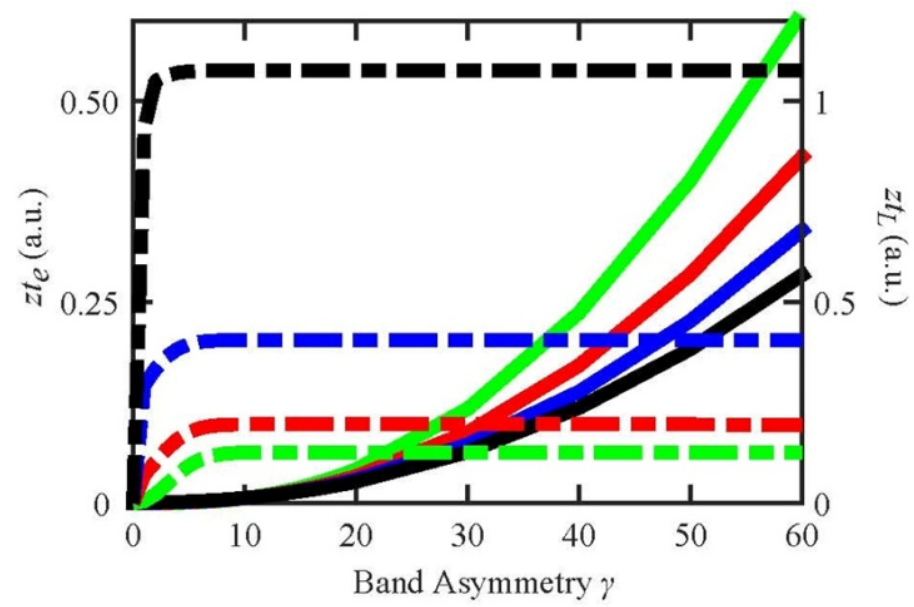

Fig. 2 Illustration of how the maximum values of $z t_{e}$ and $z t_{L}$ will be affected by the band asymmetry ratio $(\gamma)$. Without loss of generality, the reduced band gap of $\varepsilon_{g}=20$ is illustrated. The colored solid (dashed) curves represent the values $z t_{\mathrm{e}}\left(z t_{L}\right)$ for cases with different energy sensitivities $(s)$. The legend is the same as defined in Fig. 1 (a). Unlike the parameter of $s$ in Fig. 1 (a), $\gamma$ is a parameter that can increase both $z t_{e}$ and $z t_{L}$ together. In other words, the task of enhancing $Z T$ always prefers holes and electrons to be as different from each other as possible, i.e. $Z T$ benefits from large band asymmetry ratio $\gamma$.

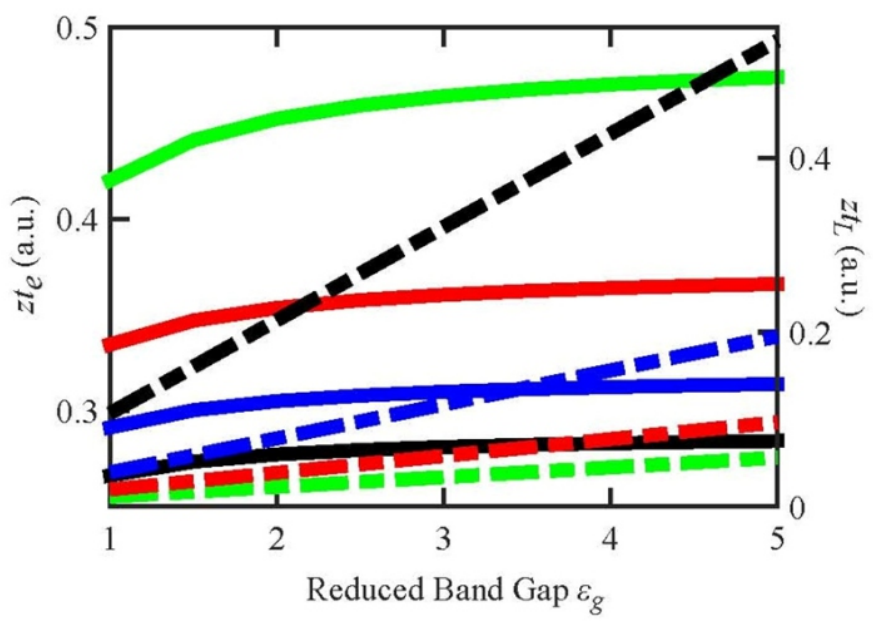

Fig. 3 Illustration of how the maximum values of $z t_{e}$ and $z t_{L}$ will be affected by the reduced band gap $\left(\varepsilon_{g}=E_{g} / k_{B} T\right)$. Without loss of generality, $\gamma$ is fixed to be 1 . The colored solid (dashed) curves represent the values $z t_{\mathrm{e}}\left(z t_{L}\right)$ for cases with different energy sensitivities as well. The legend is the same as defined in Fig. 1 (a). Contrary to conventional thought, it is shown that $\varepsilon_{g}$ will enhance $z t_{\mathrm{e}}$ and $z t_{L}$ together, though the increase of $z t_{L}$ will not be obvious when $\varepsilon_{g}$ is greater than 10 . This has dissolved the traditional "fear" of large band gap. It implies that we only need to avoid selecting large band gap materials systems, of which the thermal conductivity is too high and cannot be reduced. 
superscript ${ }^{(1)}{ }^{(2)}$ and ${ }^{(1)+(2)}$ indicates the band valley (valleys) that is (are) involved in transport, and $\max \left[z t_{\max }^{(1)}, z t_{\max }^{(2)}\right]$ means the greater value of $z t_{\max }^{(1)}$ and ${ }^{z t_{\max }^{(2)}}$. Fig. 4 (a) shows that multiple valleys will not help $z t_{e}$ : nonzero band-edge displacement $\Delta \varepsilon$ can even reduce $z t_{e}$, while band dissimilarity $\zeta$ does not influence $z t_{e}$ significantly. Fig. 4 (b) shows that multiple valleys can increases $z t_{L}$, only if the band-edge displacement $\Delta \varepsilon$ is small and the valley shape dissimilarity $\zeta$ is close to 1 . The upperlimit of the optimization ratio for $z t_{L}$ is the number of band valleys ( 2 in this case), which occurs when $\Delta \varepsilon=0$ and $\zeta=1$. This is a hint that for large carrier concentration systems, we prefer to choose materials whose band structures are with multiple similar band valleys. For small carrier concentration systems, e.g. large band gap materials, we prefer simple band structures.

In order to see how these concepts work in real materials, we have examined a range of known room temperature thermoelectric materials, of which the various properties that we need for $Z T$ estimations are well measured or calculated. From the above results, we see that the band asymmetry ratio $\gamma$ and the reduced band gap $\varepsilon_{g}$ will increase $z t_{e}$ and $z t_{L}$ together, so we now explore how these two parameters of band structure will influence the maximum $Z T$. As we know, the ultimate $Z T$ depends on many factors besides the band structure, e.g. scattering mechanisms, sample synthesis conditions, defects concentrations and grain size distributions, so we here calculate the upper limit of $Z T$ based on the commonly used acoustic phonon scattering approximation, which works well enough for the searching and screening purpose. The results of $\mathrm{ZnTe}^{43,}{ }^{44} \mathrm{SnO}_{2}{ }^{45,}{ }^{46} \mathrm{ZnO}^{47,}{ }^{48} \mathrm{ZnSe}^{49,}{ }^{50} \mathrm{CoO}$, ${ }^{52} \mathrm{Mg}_{2} \mathrm{Ge}^{53} \mathrm{Mg}_{2} \mathrm{Si}^{53}$ $\mathrm{PbS},{ }^{5456} \mathrm{NiO},{ }^{51,57,58}$ and $\mathrm{PbTe}^{59,60}$ are shown in Fig. 5, where we see that the upper limit of $Z T$ is positively correlated with $\gamma$ and $\varepsilon_{g}$, as expected from the pseudo- $Z T$ analysis above.

Finally, we show how to use the pseudo-ZT theory for searching potential materials from the band structure databases. In Fig. 6 (a)-(d), we have calculated the values of $z t_{e}$ and $z t_{L}$ at the room and the high temperatures for numerous materials with known band structures ${ }^{25}$ that are not yet well investigated for thermoelectrics. We propose that the ones with both high $z t_{e}$ and $z t_{L}$ are the most promising candidates, and deserve the priority for further studies.

In conclusion, to accelerate the search of materials candidates for thermoelectric research from the databases of calculated band structures, we have developed a pseudo-ZT framework to consider the electronic and the lattice contributions separately. Unlike $S$ and $\sigma$ or $\sigma$ and $1 / \kappa$,
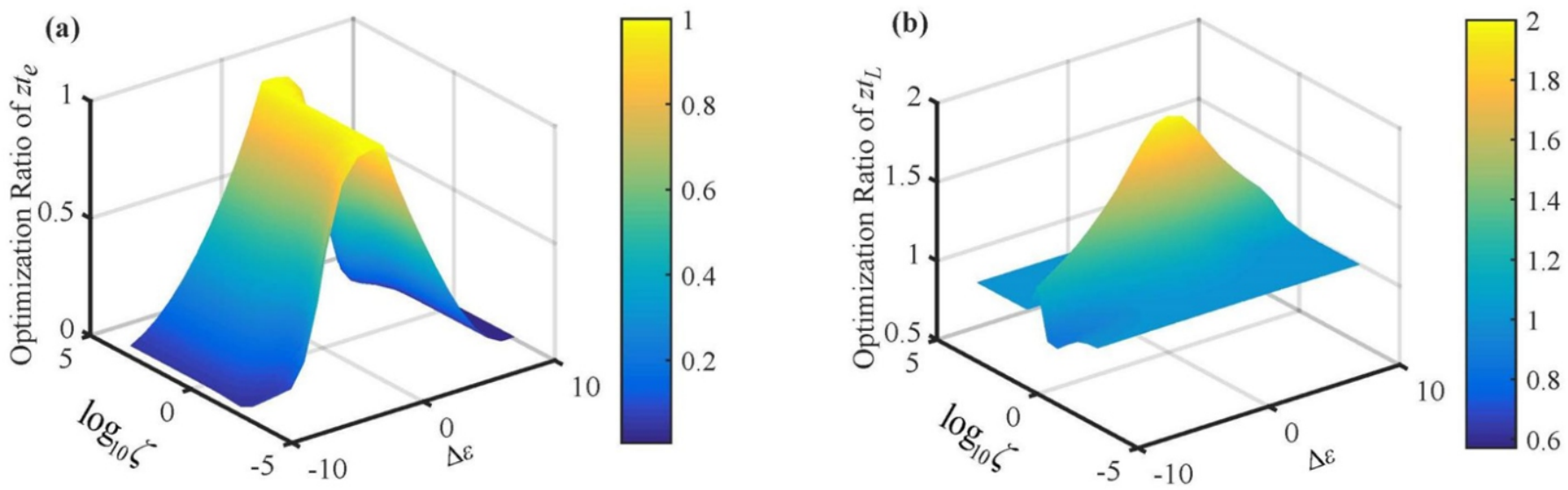

Fig. 4 Illustration of how the band alignment/degeneracy and band dissimilarity affect the optimization ratio of pseudo-ZTs defined in Equation (5). The optimization ratio of (a) $z t_{e}$ and (b) $z t_{L}$ between the hypothetic cases of having both $\mathrm{V}^{(1)}$ and $\mathrm{V}^{(2)}$ and only having $\mathrm{V}^{(1)}$ or $\mathrm{V}^{(2)}$ contributing to transport are shown as a function of the reduced band-edge displacement $\Delta \varepsilon$ and the band dissimilarity $\zeta$. Different values of $s$ give the similar trend, and we are illustrating $s=1.5$ in this figure.

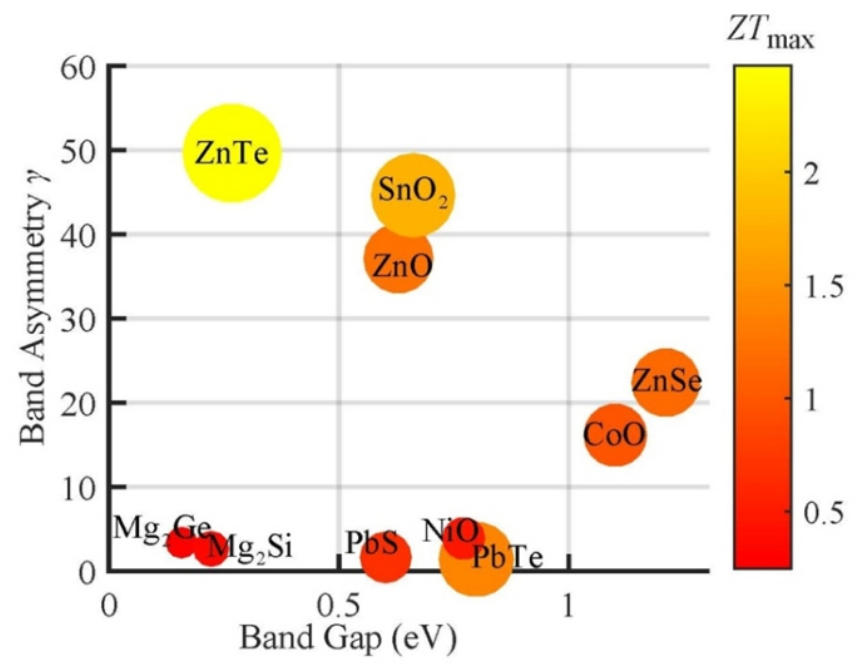

Fig. 5 The maximum $Z T$ values vs. band asymmetry ratio $(\gamma)$ and reduced band gap $\left(\varepsilon_{g}\right)$ for a range of known room temperature thermoelectric materials. Both the circle diameter and color range represent the magnitude of $Z T_{\max }$. This exhibition here has validated the beliefs based on the pseudo- $Z T$ analyses as shown in Figure 2 and 3. 
which always have a strong negative correlation, using $z t_{e}$ and $z t_{L}$ can help us avoid playing the "seesaw balancing game". In the pseudo-ZTs method, the optimization possibility of carrier scattering mechanisms and thermal conductivity will not be suppressed as in the $\beta_{S E}$ indicator method. Scenarios of both high and low carrier concentrations are considered. Furthermore, we have shown that the pseudo-ZT approach can also quantitatively reveal the physics of how the dimensionality, carrier concentration, band asymmetry, band gap and band degeneracy will affect the ultimate $Z T$. We have also shown how the upper limit of $Z T$ is influenced by the band asymmetry and band gap in several room temperature thermoelectric materials. Finally, we have carried out the calculations of pseudo-ZTs for various untested materials candidates from the band structure databases for future research guidance.

\section{Acknowledge}

This paper is in memory of Prof. Mildred Dresselhaus, who passed away on February 20, 2017. Prof. Mildred Dresselhaus made important contributions at the early stage to this paper. This work was done by S. T. partially at MIT and mainly at SUNY. This work was partially supported by AFOSR MURI Grant number FA9550-10-1-0533, subaward 60028687 and ONR MURI (OSU) grant RF01224242 for its MIT part.

\section{Competing Financial Interests}

The author(s) declare no competing interests.

\section{Author Contributions}

S. T. designed and performed the research, analyzed the data, and wrote the manuscript. M. D. contributed to improving the manuscript.

\section{References}

1. T. M. Tritt, Science, 1999, 283, 804-805.

2. G. J. Snyder and E. S. Toberer, Nat. Mater., 2008, 7, 105. (a)

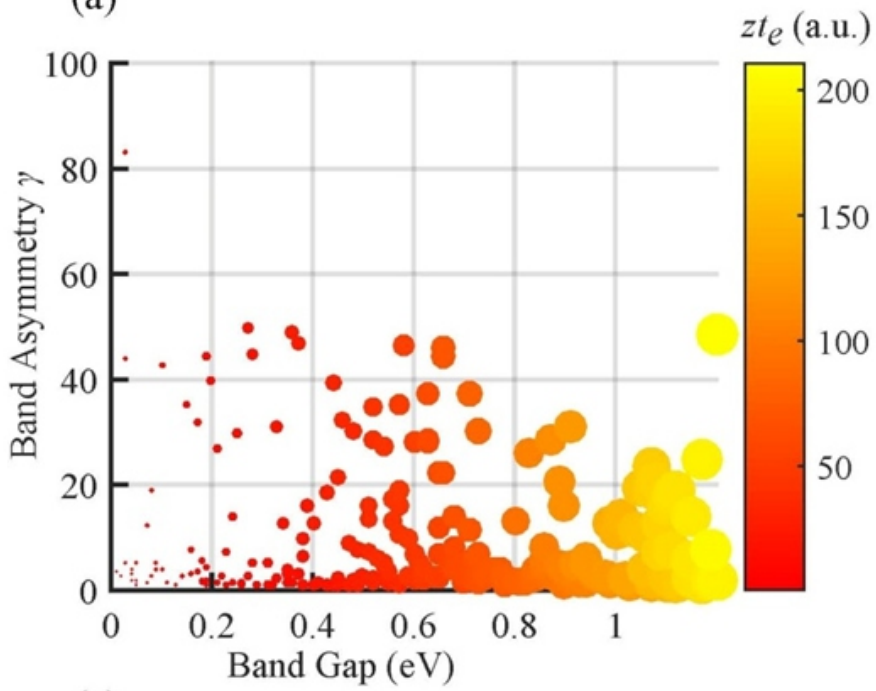

(c)

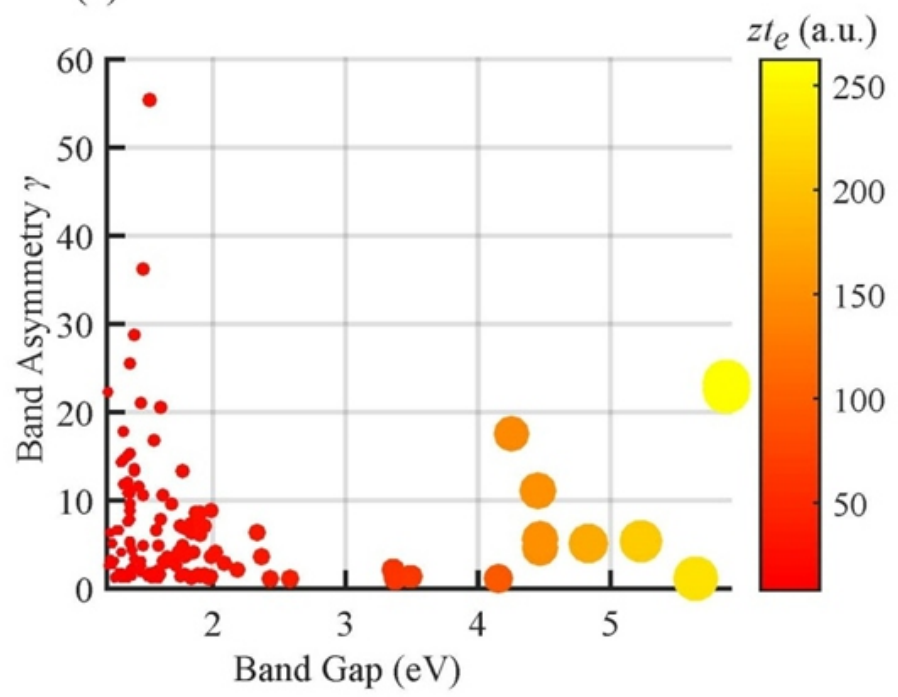

(b)

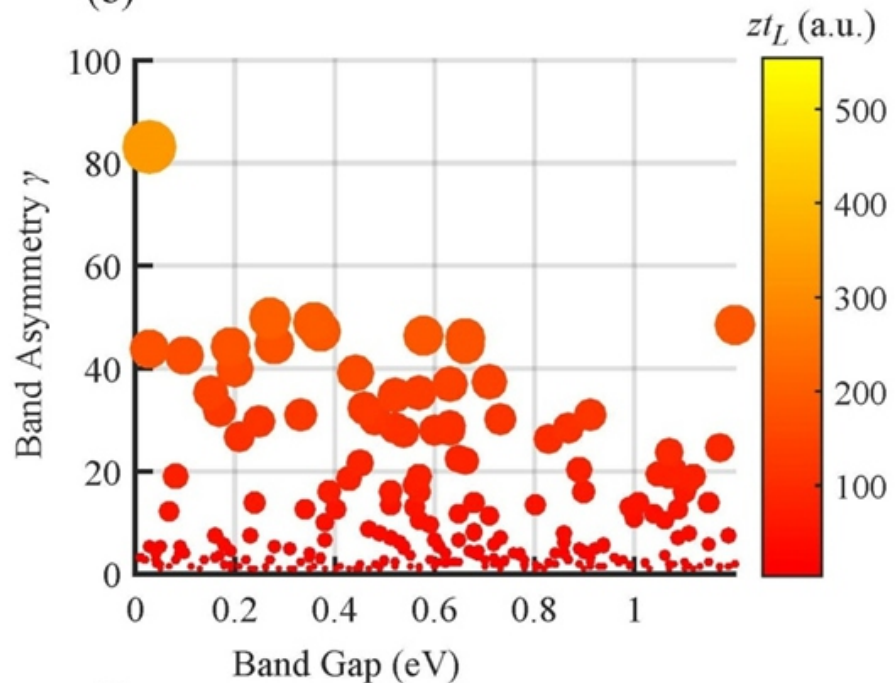

(d)

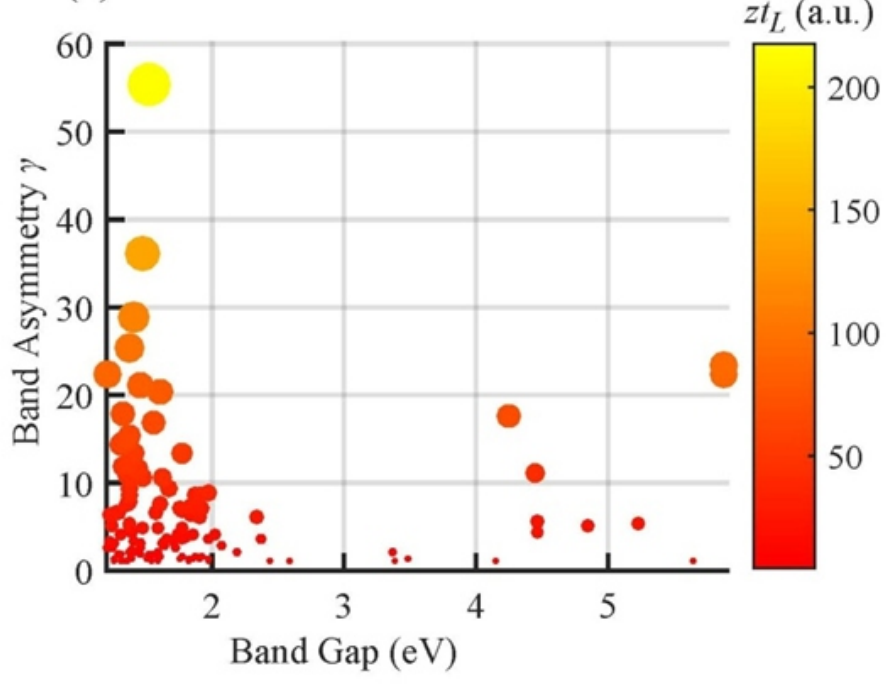

Fig. 6 Calculated (a) (c) $z t_{e}$ and (b) (d) $z t_{L}$ for materials with thermoelectric application potentials at (a) (b) room temperature (T=300 K) and (c) (d) high temperature $(T=1300 \mathrm{~K})$, of which the band structure information can be found in materials databases ${ }^{25}$. Both the circle diameter and color range represent the magnitude of pseudo-ZTs. According to our pseudo-ZT analyses in the present work, the materials with both large $z t_{e}$ and $z t_{L}$ values are the most promising candidates that should be studied with priority. 
3. M. Zebarjadi, K. Esfarjani, M. Dresselhaus, Z. Ren and G. Chen, Energy Environ. Sci., 2012, 5, 5147-5162.

4. N. Lu and I. Ferguson, Semicond. Sci. Technol., 2013, 28, 074023.

5. X. Y. Mi, X. Yu, K. L. Yao, X. Huang, N. Yang and J. T. Lü, Nano lett., 2015, 15, 5229-5234

6. D. Ma, H. Ding, H. Meng, L. Feng, Y. Wu, J. Shiomi and N. Yang, Phys. Rev. $B, 2016,94,165434$.

7. Z. Ding, M. An, S. Mo, X. Yu, Z. Jin, Y. Liao, K. Esfarjani, J. T. Lü, J. Shiomi and N. Yang, J. Mater. Chem. A, 2019, 7, 2114-2121.

8. Q. Hao, D. Xu, N. Lu and H. Zhao, Phys. Rev. B, 2016, 93, 205206.

9. L. Hicks and M. S. Dresselhaus, Phys. Rev. B, 1993, 47, 12727.

10. G. Mahan and J. Sofo, P. Natl. A. Sci., 1996, 93, 7436-7439.

11. R. Venkatasubramanian, Semiconduct. Semimet., 2001, 71, 175-201.

12. T. Harman, P. Taylor, M. Walsh and B. LaForge, Science, 2002, 297, 22292232.

13. Y. M. Lin and M. Dresselhaus, Phys. Rev. B, 2003, 68, 075304.

14. J. P. Heremans, V. Jovovic, E. S. Toberer, A. Saramat, K. Kurosaki, A. Charoenphakdee, S. Yamanaka and G. J. Snyder, Science, 2008, 321, 554557.

15. Q. Zhang, H. Wang, W. Liu, H. Wang, B. Yu, Q. Zhang, Z. Tian, G. Ni, S. Lee and K. Esfarjani, Energy \& Environmental Science, 2012, 5, 5246-5251.

16. B. Poudel, Q. Hao, Y. Ma, Y. Lan, A. Minnich, B. Yu, X. Yan, D. Wang, A. Muto and D. Vashaee, Science, 2008, 320, 634-638.

17. K. Biswas, J. He, I. D. Blum, C.-I. Wu, T. P. Hogan, D. N. Seidman, V. P. Dravid and M. G. Kanatzidis, Nature, 2012, 489, 414.

18. D. Parker, X. Chen and D. J. Singh, Phys. Rev. Lett., 2013, 110, 146601.

19. P. Hohenberg and W. Kohn, Phys. Rev., 1964, 136, B864.

20. G. Bergerhoff, R. Hundt, R. Sievers and I. Brown, J. Chem. Inf. Comp. Sci., 1983, 23, 66-69.

21. A. Belsky, M. Hellenbrandt, V. L. Karen and P. Luksch, Acta Crystallogr. B, 2002, 58, 364-369.

22. A. Jain, G. Hautier, C. J. Moore, S. Ping Ong, C. C. Fischer, T. Mueller, K. A. Persson and G. Ceder, Comp. Mater. Sci., 2011, 50, 2295-2310.

23. H. J. Goldsmid and R. W. Douglas, Brit. J. Appl. Phys., 1954, 5, 386.

24. R. P. Chasmar and R. Stratton, J. Electron. Cont., 1959, 7, 52-72.

25. J. Yan, P. Gorai, B. Ortiz, S. Miller, S. A. Barnett, T. Mason, V. Stevanovic and E. S. Toberer, Energy Environ. Sci., 2015, 8, 983-994.

26. M. Zebarjadi, K. Esfarjani, Z. Bian and A. Shakouri, Nano Lett., 2011, 11, 225-230.

27. R. J. Mehta, Y. Zhang, H. Zhu, D. S. Parker, M. Belley, D. J. Singh, R. Ramprasad, T. Borca-Tasciuc and G. Ramanath, Nano Lett., 2012, 12, 45234529

28. S. I. Kim, K. H. Lee, H. A. Mun, H. S. Kim, S. W. Hwang, J. W. Roh, D. J. Yang, W. H. Shin, X. S. Li, Y. H. Lee, G. J. Snyder and S. W. Kim, Science, 2015, 348, 109-114.

29. L. Lindsay, D. A. Broido and T. L. Reinecke, Phys. Rev. Lett., 2012, 109, 095901.

30. L. D. Zhao, S. H. Lo, Y. Zhang, H. Sun, G. Tan, C. Uher, C. Wolverton, V. P. Dravid and M. G. Kanatzidis, Nature, 2014, 508, 373.
31. K. Esfarjani, G. Chen and H. T. Stokes, Phys. Rev. B, 2011, 84, 085204.

32. J. O. Sofo and G. D. Mahan, Appl. Phys. Lett., 1994, 65, 2690-2692.

33. S. Tang and M. S. Dresselhaus, Appl. Phys. Lett., 2014, 105, 033907.

34. V. J., S. Ali, M. Arun and K. M. G., Adv. Mater., 2010, 22, 3970-3980.

35. M. G. Kanatzidis, Chem.Mater., 2010, 22, 648-659.

36. M. S. Dresselhaus, G. Chen, Z. F. Ren, G. Dresselhaus, A. Henry and J. P. Fleurial, JOM, 2009, 61, 86-90.

37. D. M. Rowe, Modules, Systems, and Applications in Thermoelectrics, Taylor \& Francis, 2012.

38. D. Hsieh, D. Qian, L. Wray, Y. Xia, Y. S. Hor, R. J. Cava and M. Z. Hasan, Nature, 2008, 452, 970.

39. L. Fu, C. L. Kane and E. J. Mele, Physical Review Letters, 2007, 98, 106803. 40. S. Tang and M. S. Dresselhaus, Nano Lett., 2012, 12, 2021-2026.

41. S. Tang and M. S. Dresselhaus, Nanoscale, 2012, 4, 7786-7790.

42. Y. Pei, A. LaLonde, S. Iwanaga and G. J. Snyder, Energy Environ. Sci., 2011, 4, 2085-2089.

43. K. Davami, A. Weathers, N. Kheirabi, B. Mortazavi, M. T. Pettes, L. Shi, J. S. Lee and M. Meyyappan, J. Appl. Phys., 2013, 114, 134314.

44. H. Wang and W. Chu, J. Alloy. Compd., 2009, 485, 488-492.

45. P. Turkes, P. Ch and R. Helbig, J. Phys. C: Sol. State Phys., 1980, 13, 4941.

46. B. P. R., V. J. A., B. C. M., L. E. and L. E. R., J. Am. Ceram. Soc., 2005, 88, 2629-2631.

47. Y. Xu, M. Goto, R. Kato, Y. Tanaka and Y. Kagawa, J. Appl.Phys., 2012, 111, 084320 .

48. X. Wu, J. Lee, V. Varshney, J. L. Wohlwend, A. K. Roy and T. Luo, Sci. Rep$U K, 2016,6,22504$

49. N. V. Lugueva, S. M. Luguev and A. A. Dunaev, Phys. Sol. State, 2003, 45, $449-452$

50. B. A. K., S. N., R. S. K. and R. K., Cryst. Res. Technol., 2004, 39, 558-563.

51. F. B. Lewis and N. H. Saunders, J. Phys. C: Sol. State Phys., 1973, 6, 2525.

52. M. Massot, A. Oleaga, A. Salazar, D. Prabhakaran, M. Martin, P. Berthet and G. Dhalenne, Phys. Rev. B, 2008, 77, 134438

53. J. J. Martin, J. Phys. Chem. Sol., 1972, 33, 1139-1148.

54. J. L. Wang, H. Wang, G. J. Snyder, X. Zhang, Z. H. Ni and Y. F. Chen, J. Physics D: Appl. Phys., 2013, 46, 405301.

55. D. Greig, Phys. Rev., 1960, 120, 358-365.

56. L. Wei, J. F. Chen, Q. Y. He and W. Teng, J.Alloy. Compd., 2014, 584, 381384.

57. P. Sahoo, D. Misra, G. Shankar Chaubey, J. Salvador, N. J. Takas and P. F. P. Poudeu, Thermal Conductivity of Nickel Oxide Nanoparticles Synthesized by Combustion Method, 2011.

58. P. Sahoo, D. K. Misra, J. Salvador, J. P. A. Makongo, G. S. Chaubey, N. J. Takas, J. B. Wiley and P. F. P. Poudeu, J.Sol. State Chem., 2012, 190, 29-35.

59. D. T. Morelli, V. Jovovic and J. P. Heremans, Phys. Rev. Lett., 2008, 101, 035901.

60. A. H. Romero, E. K. U. Gross, M. J. Verstraete and O. Hellman, Phys. Rev. $B, 2015,91,214310$

Publisher's Note Engineered Science Publisher remains neutral with regard to jurisdictional claims in published maps and institutional affiliations. 